\title{
A Unified Management for Spontaneous CSF Leak
}

\author{
Lobna El Fiky1, Ali Kotb², Badr Eldin Mostafa1 \\ ${ }^{1}$ Otolaryngology Department, Ain Shams University, Cairo, Egypt \\ ${ }^{2}$ Neurosurgery Department, Ain Shams University, Cairo, Egypt \\ Email: elfikylobna@hotmail.com, elfikylobna@gmail.com
}

Received 27 February 2015; accepted 15 March 2015; published 19 March 2015

Copyright (C) 2015 by authors and Scientific Research Publishing Inc.

This work is licensed under the Creative Commons Attribution International License (CC BY). http://creativecommons.org/licenses/by/4.0/

(c) (i) Open Access

\section{Abstract}

Background: Spontaneous CSF leak represents less frequent cause of CSF leak, but cases are more difficult to control, with the highest failure rate and recurrence despite adequate repair. The problems in these cases might be related to an underlying undiagnosed associated intracranial hypertension. Recognition and long-term treatment of elevated ICP is therefore critical to the successful management of these patients. Objective: To evaluate the CSF pressure in cases of spontaneous CSF rhinorrhea and to describe our same setting combined protocol to the repair of the leak, measurement and management of CSF pressure. Patients and Methods: All patients presenting to Ain Shams University Hospitals, Cairo, Egypt, with spontaneous CSF leak were included prospectively in the study. Clinical and radiologic data were collected to suspect elevated intracranial pressure. After CSF repair, CSF pressure was measured and if found to be more than $20 \mathrm{cmH}_{2} \mathbf{O}$, a lumboperitoneal shunt was used. Results: Twenty-seven cases, 23 women and 4 men, presented with spontaneous CSF leak. 23 patients had BMI above 30. All patients had empty sella syndrome $(100 \%)$, and a meningoencephalocele was found in 13 cases (48\%). CSF pressure ranged from 5 to 39 $\mathrm{cmH}_{2} \mathrm{O}$ (mean $=28.7$ ). A pressure above $21 \mathrm{cmH}_{2} \mathrm{O}$ was found in19 patients $(70 \%)$ and subsequently had lumboperitoneal shunt in the same setting. No recurrence occurred in this subset of patients with 6 - 60 months follow-up period. Conclusion: A selective and specific same setting protocol can result in a better diagnosis and control of the accompanying elevated ICP in cases of spontaneous CSF leak. It avoids a second operative intervention, and shortens the hospital stay, with an increase in the success rate. In the same time, the smaller number of patients with normal ICP can avoid further drainage.

\section{Keywords}

CSF Rhinorrhea, Elevated ICP, Spontaneous CSF Leak, Lumbar Drain, Acetazolamide, Empty Sella Syndrome, Intracranial Hypertension, Endoscopic CSF Repair, CSF Diversion, Lumboperitoneal Shunts 


\section{Introduction}

Spontaneous CSF leak represents a distinct clinical entity that warrants a separate designation [1]. Although less frequent than other causes of CSF leak, cases are more difficult to control, with the highest failure rate and recurrence despite adequate repair [2].

Although the precise cause and mechanism of spontaneous CSF leaks are not fully understood, the knowledge and experience with spontaneous CSF leaks have increased dramatically over the last decade [3]. The problems in these cases might be related to an underlying undiagnosed associated intracranial hypertension and its correction may be warranted [4]-[8].

The majority of these patients exhibit demographics, clinical symptoms and radiographic signs of elevated intracranial pressure (ICP) and should be considered as a high-pressure condition [1] [3] [5] [9]-[12]. Similarities include middle age obese females and empty sella [13]. Direct evidence of elevated ICP in most patients with spontaneous CSF leaks was also found [3] [7] [13] [14].

The pressure usually exceeds $20 \mathrm{cmH}_{2} \mathrm{O}$ fitting with modified Dandy's criteria for intracranial hypertension [1]. The high ICP has been identified as a negative risk factor for successful repair [2] [15]. However, the raised ICP does not become evident until the pressure is rechecked after the leak is closed [16].

Worsening of pressure-type headaches and pulsatile tinnitus can occur after successful repair of CSF leak probably due to elimination of nasal CSF diversion via the leak and the resultant rise in the ICP post-operatively [5].

Several reports initiated a protocol to prospectively identify and treat patients with CSF leaks and elevated ICP and therefore at risk for recurrence of the leak [17]. Recognition and long-term treatment of elevated ICP is therefore critical to the successful management of these patients.

The purpose of our study was to evaluate the CSF pressure in cases of spontaneous CSF rhinorrhea and to report the outcome of repair of these cases when the elevated ICP was addressed. We also describe our same setting combined protocol to the repair of the leak, measurement and management of CSF pressure in this subset of patients.

\section{Materials and Methods}

In this prospective descriptive study, all patients presenting with primary or recurrent spontaneous CSF leak, to Ain Shams University Hospitals, Otolaryngology and Neurosurgical Departments between September 2008 and September 2013 were included. Patients with traumatic or iatrogenic causes were excluded. This study was approved by the Ethical committee for Ain Shams University, Cairo, Egypt and all patients signed a written consent for inclusion.

All demographic data, clinical presentation, body mass index (BMI), comorbidities and duration of leak were collected as well as chemical confirmation of the leaked fluid. All patients had visual acuity and fundus examination evaluation. Radiological workup included a CT scan paranasal sinuses without contrast, thin cuts, bony window, axial and coronal views in addition to an MRI, T1W, T1Gd and heavily weighted T2W sequences to localize the leak in the nasal cavity. A CT scan with metrizamide was also done for all patients.

The protocol for treatment included: decision on route of access, whether endoscopic, external or cranial approach; measurement of CSF pressure after completion of the repair; insertion of a lumboperitoneal shunt if the pressure exceeded $20 \mathrm{cmH}_{2} \mathrm{O}$ at the end of the operation.

All patients were discharged within 48 - 72 hours postoperatively with a Merocel pack in place and instructions to avoid increase intracranial pressure. The pack was removed on the 5th day in the outpatient clinic.

A routine paranasal sinus CT scan and abdominal plain X-ray were performed the next day of the operation. Patients with an intraoperative CSF pressure of $11-19 \mathrm{cmH}_{2} \mathrm{O}$, had a re-measuring of the pressure 3 days after operation and if the pressure was still high a shunt was immediately performed (Figure 1). All patients were followed up and all postoperative complications were documented.

\section{Results}

Twenty-seven cases presented with spontaneous CSF leak during the study period. They were 23 women and 4 men. Their age ranged from 29 to 68 years old (mean $=45.3$ years). Their BMI ranged from 26.6 to 43 (mean = 38.5). Four patients had a BMI of 25 - 30, 9 patients had a BMI between 31 and 35 and 14 patients had a BMI above 36. Five patients had hypertension, while 2 patients were diabetic. Four patients had recurrent leak after previous endoscopic repair. Most of the patients either received or were intolerant to acetazolamide treatment 


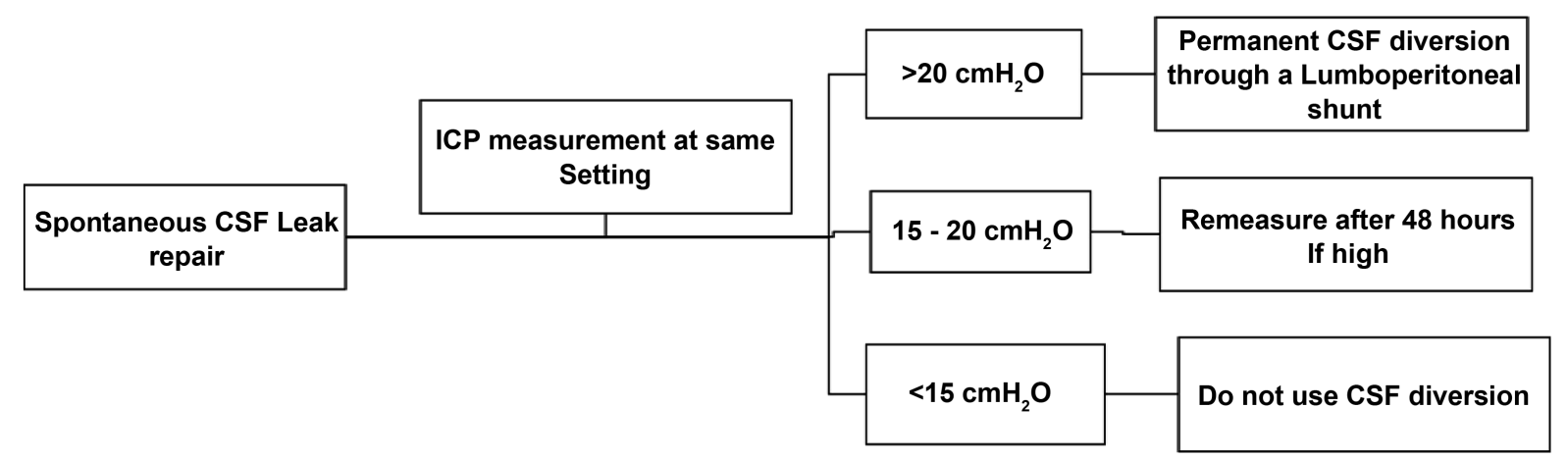

Figure 1. Protocol for management of spontaneous CSF leak and ICP.

before presenting to us without response.

The site of the leak was localized preoperatively in the radiological examination in all cases (Table 1). No cases had multiple sites of leak in our series. A meningoencephalocele was found in 13 cases (48\%) (Figure 2).

The radiological data suspicious of increased intracranial pressure were looked for in CT scan and MRI. All patients had empty sella syndrome (100\%) whether partial or complete (Figure 3). Ten patients had slit-like ventricles and tight subarachnoid spaces, while only 5 patients had tortuous optic nerve (Figure 4).

Surgery: All the repairs were performed extracranially: Endoscopic repair in 24 cases and external frontoethmoidectomy approach in 3 cases for a defect in the posterior table of the frontal sinus. The same technique was used in all cases for repair: underlay and overlay fascia lata graft with tissue glue use. Gel foam packing and Merocel $^{\circledR}$ nasal pack were further used. In large size defects more than $1.5 \mathrm{~cm}$, a septal cartilage graft was also added.

At the end of the procedure, after graft placement and nasal packing, the patient was repositioned in the lateral decubitus and CSF pressure measurement was done using a wide bore lumbar drain needle. A collecting chamber with a pressure transducer was connected to the drain and zeroed at the spinal cord and the pressure was read when the flow stabilized. CSF pressure ranged from 5 to $39 \mathrm{cmH}_{2} \mathrm{O}$ (mean = 28.7). Only 2 patients had a pressure below $10 \mathrm{cmH}_{2} \mathrm{O}$ (7\%), 6 had pressure between $11-20 \mathrm{cmH}_{2} \mathrm{O}$ (22\%) and 19 patients (70\%) had a pressure above $21 \mathrm{cmH}_{2} \mathrm{O}$.

A lumboperitoneal shunt (Johnson $\left.{ }^{\circledR} 84 \mathrm{~cm}\right)$ was inserted in all patients with a pressure $>21 \mathrm{cmH}_{2} \mathrm{O}(\mathrm{n}=19)$. These patients stayed $48-72$ hours in the hospital and were then discharged with the nasal pack in situ. Patients with a pressure between 11 and $20 \mathrm{cmH}_{2} \mathrm{O}(\mathrm{n}=6)$ had a repeat measurement 3 days post-operatively. Only 2 patients had a pressure above $20 \mathrm{cmH}_{2} \mathrm{O}$ and had the shunt inserted 4 days after the operation. The remaining 4 patients had a lower CSF pressure and had no further interventions and were discharged accordingly.

Complications: Minimal pneumocephalus occurred in 2 cases and resolved spontaneously without neurological affection. Postoperative headache for up to one month occurred in 6 cases. Those patients received Acetazolamide $500 \mathrm{mg}$ twice daily for at least one month for postoperative headache development.

There were 5 cases of intranasal adhesions, 2 cases of abdominal wound gaping, one case of incisional hernia, and one case of shunt infection necessitating removal after 2 months.

A delayed frontal mucocele occurred in a patient treated externally after 8 months necessitating reoperation. All patients had successful repair with no recurrences with follow up period of $6-60$ months (mean $=30$ months) (Figures 2-5).

\section{Discussion}

Spontaneous CSF leak is a challenging clinical condition, with frequent recurrences following attempted surgical closure [12]. These recurrences should be considered as failure of management of elevated ICP rather than failure of operative repair 3. Decreasing ICP may have a beneficial adjuvant role to endoscopic repair of CSF rhinorrhea. Better management of the elevated ICP in this specific group of patients may lead to higher success rates [2]. The art of repairing these defects lies in knowing which patients will require CSF diversion and which who would not need it.

Patients with elevated ICP should preferably be identified upfront avoiding a second operation [17]. Although 
Table 1. Preoperative localization of the site of leak.

\begin{tabular}{ccc}
\hline Site of leak & Number \\
\hline Cribriform plate & 8 \\
Fovea ethmoidalis & 5 \\
Cribriform + fovea & 5 \\
Frontal sinus & 4 \\
Sphenoid sinus & 4 \\
Sellaturcica & 1 \\
\hline
\end{tabular}

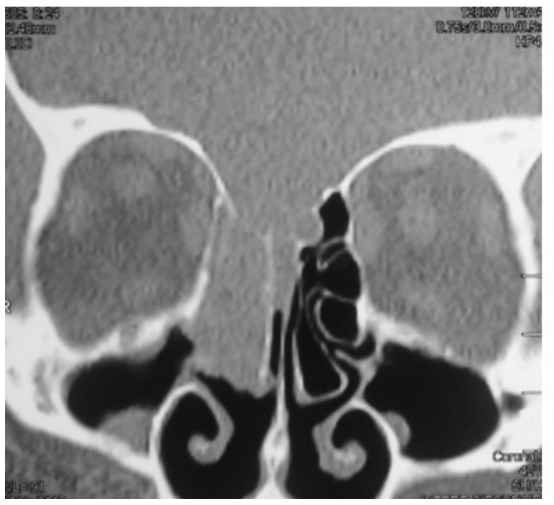

(a)

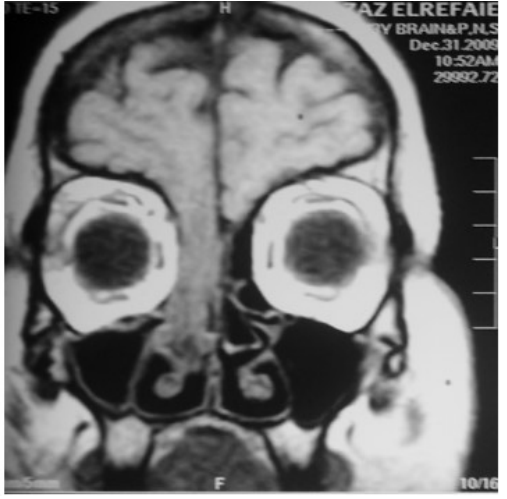

(b)

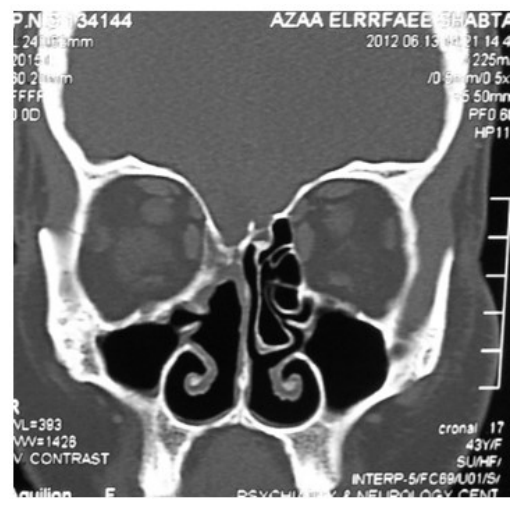

(c)

Figure 2. (a) Coronal CT scan PNS; (b) Coronal T1 weighted MRI showing a right sided huge meningoencephalocele, involving right fovea ethmoidalis and cribrifom plate; (c) Follow up CT scan 12 months after the repair showing success of the repair.

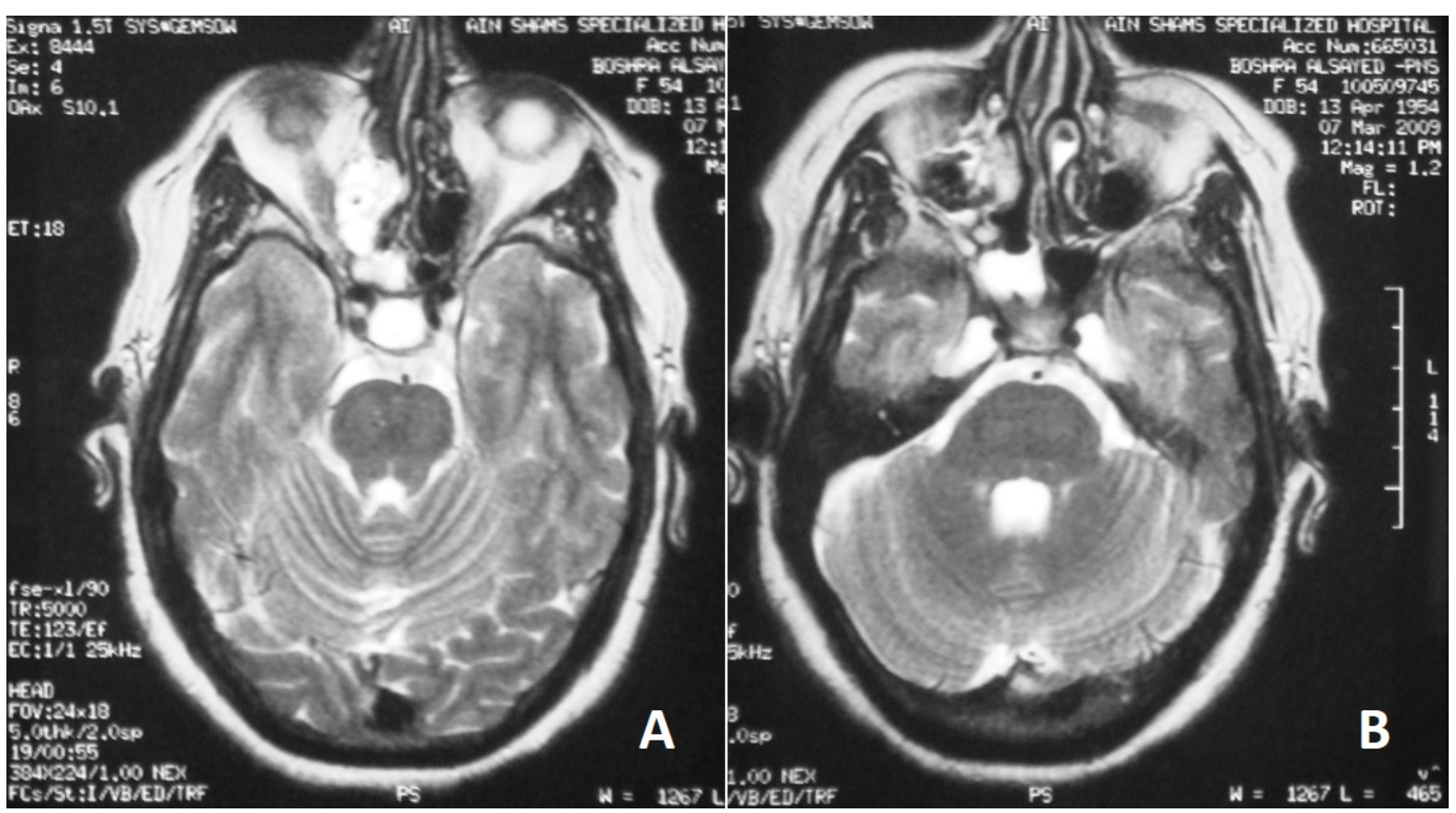

(a)

(b)

Figure 3. Axial T2 weighted MRI of paranasal sinuses showing (a) empty sella syndrome; bilaterally distended Meckel's cave (b). CSF leak is seen in the right ethmoid air cells and nasal cavity (a) \& (b). 


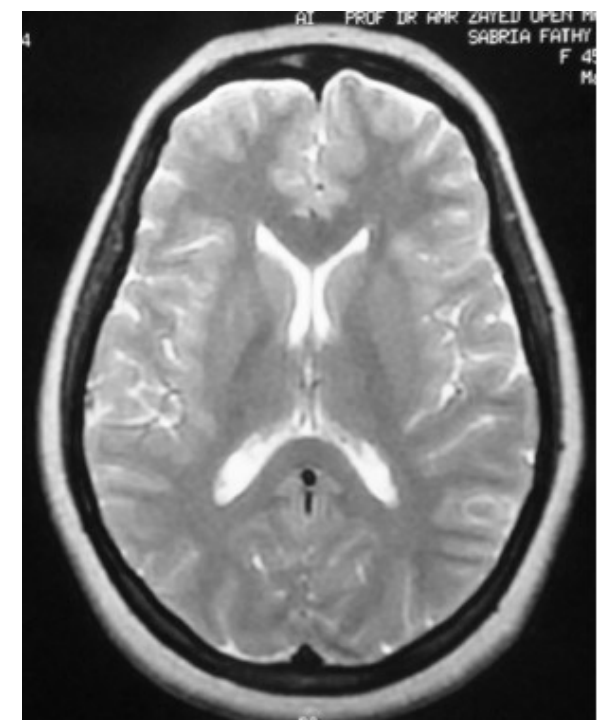

(a)

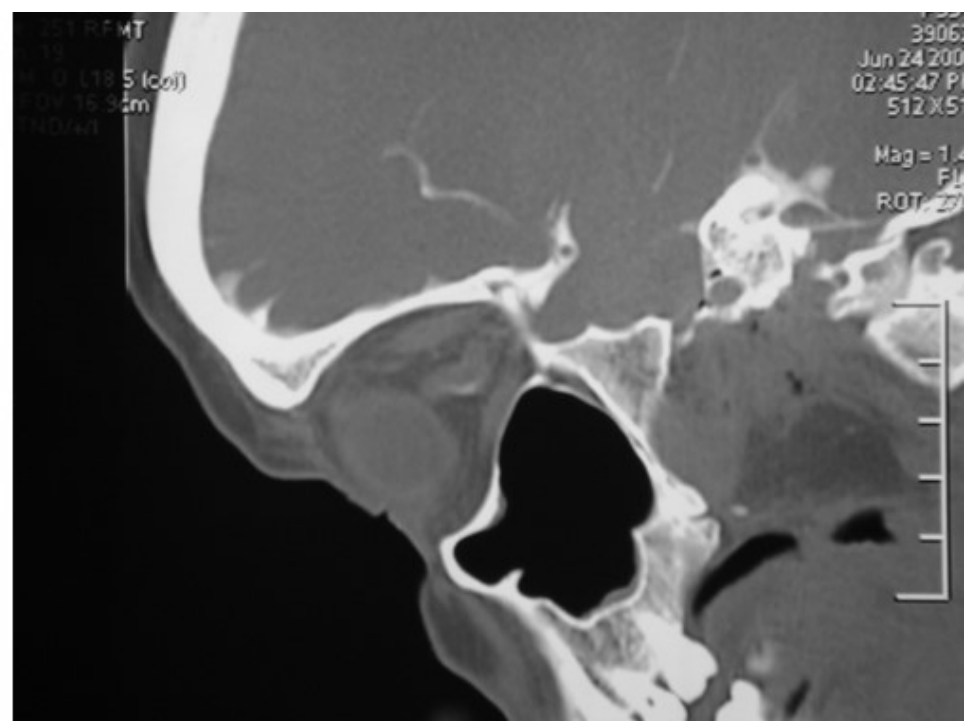

(b)

Figure 4. Radiologic criteria of elevated ICP (a) T2 weighted MRI showing slit-like ventricles and tight subarachnoid spaces (b) Sagittal CT scan showing tortuous optic nerve.

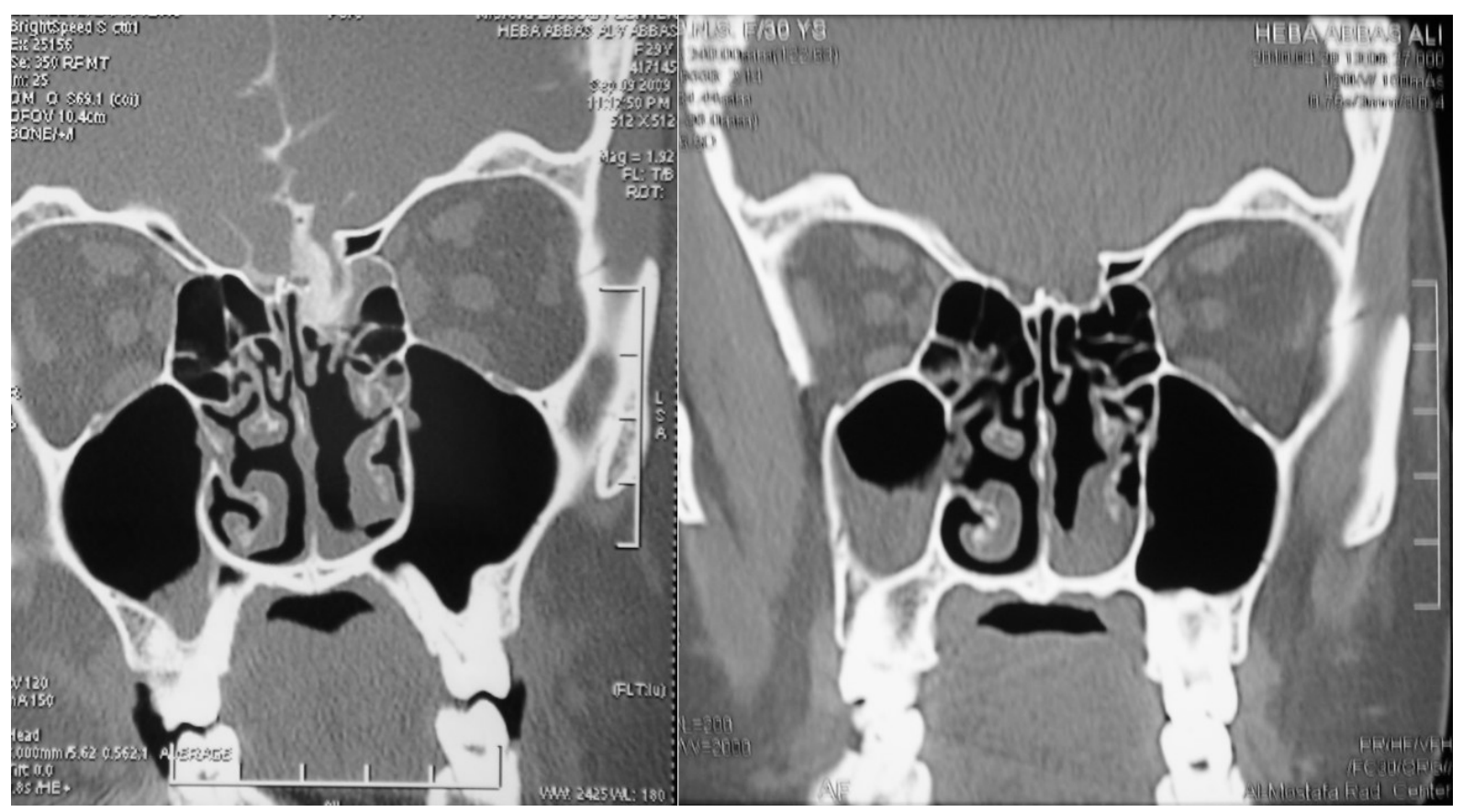

(a)

(b)

Figure 5. (a) CT cisternography with metrizamide showing a left sided large skull base defect in the fovea ethmoidalis; (b) Follow up CT scan after 12 months showing successful repair.

there are several clinical and radiologic clues, there are no rules [18]. Regarding the clinical clues, most of patients were females and BMI was above 30.

Radiologic findings may serve as a non-invasive indicator of elevated ICP. Radiographic evidence of elevated ICP include, but not limited to, small ventricles, a partial or complete empty sella, formation of arachnoid pits, abnormalities of the optic nerve sheath complex and meningoencephaloceles [1] [2] [4] [13] [15] [19]-[22]. Regarding radiologic clues in our patients, several were found especially the empty sella syndrome.

The pressure can be directly measured at the time of operation or post-operatively. Whenever the ICP is high, 
several authors resort to temporary or permanent CSF lowering. This can be achieved temporarily via medical treatment with acetazolamide, or through a lumbar drain [12] [23] [24]. However, inadequate response or intolerance to acetazolamide has been increasingly documented. A large number of our cases received prolonged treatment before presenting to us with no control. Lumbar drains have been advocated to transiently reduce CSF pressure in the immediate postoperative period and may also serve to determine which patients require longterm management of increased ICP2. Little is known about the indications or efficacy of post-operative lumbar drains to control CSF pressure after endoscopic repair of CSF rhinorrhea and its use remains controversial [24] [25]. Most authors use a lumbar drain during surgery and maintain it in the postoperative period for CSF diversion and measurement [5] [13] [17] [18]. If CSF pressure is high they recommend medical treatment but if the pressure is higher than 35 they consider permanent shunting [5].

However, not only is there a potential for associated complications, but there is also the need for hospitalization when these drains are used [26]. These are the same complications occurring when using a permanent lumboperitoneal shunt, and we find the benefits far outweigh the risks of such a minor procedure in well trained hands. In the same time, cases with refractory elevated ICP to a combination of medical management by acetazolamide and lumbar drain in spontaneous CSF leak has been shown to occur [14]. These patients usually have delayed recurrent leakage that may require permanent diversion procedures in a revision setting [5] [16]. This failure usually occurs later than 14 months, which implies the need of a long-term solution rather than a temporary one.

In our protocol, we prefer to measure the CSF pressure at the end of the repair, after depletion of the CSF during the repair. If the pressure is still high, we resort to permanent CSF diversion through a lumboperitoneal shunt in the same setting, sparing a second operation, allowing early ambulance, shortening hospital stay, increasing the success rate of the graft and decreasing recurrence rate. However, shunts are not without associated morbidity, so careful patient selection should be considered [17]. Those were mild and temporary in our group of patients. In our series, $70 \%$ of the cases had a high ICP above $21 \mathrm{cmH}_{2} \mathrm{O}$, which can constitute a part of the pathophysiology. However, the smaller number without elevation of ICP were spared from a permanent CSF diversion.

Our $100 \%$ success rate of CSF leak control is consistent with previous reports. We believe that the high rate of success in this subset of patients, is basically related to the ability to control their ICP.

This approach is properly tailored to the individual patient, addressing both the elevated ICP and CSF leak in a single setting. Using this selective and specific protocol, a better diagnosis and control of the accompanying elevated ICP, when present, can be achieved. This avoids a second operative intervention, shortens the hospital stay, with an increase in the success rate. In the same time, the smaller number of patients with normal ICP can avoid further drainage.

\section{Conclusions}

We emphasize that management of elevated CSF in spontaneous CSF leak patients should be considered as an integral part of the control of the leak and prevent its recurrence.

In the cases with proved elevated ICP preoperatively by demographic, clinical, and radiological criteria as well as by measuring of CSF pressure at the end of the repair, permanent CSF diversion in the same setting should be considered. Further prospective case control studies are definitely needed to determine the need for long-term CSF diversion in that specific group of patients.

\section{References}

[1] Schlosser, R. and Bolger, W. (2003) Significance of Empty Sella in Cerebrospinal Fluid Leaks. Otolaryngology —Head and Neck Surgery, 128, 32-38. http://dx.doi.org/10.1067/mhn.2003.43

[2] Wang, E.W., Vandergrift III, W.A. and Schlosser, R.J. (2011) Spontaneous CSF Leaks. Otolaryngologic Clinics of North America, 44, 845-856. http://dx.doi.org/10.1016/j.otc.2011.06.018

[3] Woodworth, B.A., Prince, A., Chiu, A.G., Cohen, N.A., Schlosser, R.J., Bolger, W.E., Kennedy, D.W. and Palmer, J.N. (2008) Spontaneous CSF Leaks: A Paradigm for Definitive Repair and Management of Intracranial Hypertension. Otolaryngology—Head and Neck Surgery, 138, 715-720. http://dx.doi.org/10.1016/j.otohns.2008.02.010

[4] Woodworth, B., Bolger, W. and Schlosser, R. (2006) Nasal Cerebrospinal Fluid Leaks and Encephaloceles. Operative Techniques in Otolaryngology, 17, 111-116. http://dx.doi.org/10.1016/j.otot.2006.03.001

[5] Schlosser, R.J., Wilensky, E.M., Grady, M.S., Palmer, J.N., Kennedy, D.W. and Bolger, W.E. (2004) Cerebrospinal 
Fluid Pressure Monitoring after Repair of Cerebrospinal Fluid Leaks. Otolaryngology—Head and Neck Surgery, 130, 443-448. http://dx.doi.org/10.1016/j.otohns.2003.12.018

[6] Schlosser, R.J. and Bolger, W.E. (2002) Management of Multiple Spontaneous Nasal Meningoencephaloceles. Laryngoscope, 112, 980-985. http://dx.doi.org/10.1097/00005537-200206000-00008

[7] Schlosser, R.J., Maloney-Wilensky, E., Grady, M.S., et al. (2003) Elevated Intracranial Pressures in Spontaneous Cerebrospinal Fluid Leaks. American Journal of Rhinology, 17, 191-196.

[8] Schlosser, R.J. and Bolger, W.E. (2004) Nasal Cerebrospinal Fluid Leaks: Critical Reviews and Surgical Considerations. Laryngoscope, 114, 255-265. http://dx.doi.org/10.1097/00005537-200402000-00015

[9] Schlosser, R.J. and Bolger, W.E. (2006) Spontaneous Cerebrospinal Fluid Leaks: A Variant of Benign Intracranial Hypertension. Annals of Otology, Rhinology, and Laryngology, 115, 495-500. http://dx.doi.org/10.1177/000348940611500703

[10] Pérez, M.A., Bialer, O.Y., Bruce, B.B., Newman, N.J. and Biousse, V. (2013) Primary Spontaneous Cerebrospinal Fluid Leaks and Idiopathic Intracranial Hypertension. Journal of Neuro-Ophthalmology, 33, 330-337. http://dx.doi.org/10.1097/WNO.0b013e318299c292

[11] Chaaban, M.R., Illing, E., Riley, K.O. and Woodworth, B.A. (2014) Spontaneous Cerebrospinal Fluid Leak Repair: A Five-Year Prospective Evaluation. Laryngoscope, 124, 70-75. http://dx.doi.org/10.1002/lary.24160

[12] Woodworth, B.A. and Palmer, J.N. (2009) Spontaneous Cerebrospinal Fluid Leaks. Current Opinion in Otolaryngology \& Head \& Neck Surgery, 17, 59-65. http://dx.doi.org/10.1097/MOO.0b013e3283200017

[13] Schlosser, R.J., Wilensky, E.M., Grady, M.S., Palmer, J.N., Kennedy, D.W. and Bolger, W.E. (2004) Cerebrospinal Fluid Pressure Monitoring after Repair of Cerebrospinal Fluid Leaks. Otolaryngology—Head and Neck Surgery, 130, 443-448. http://dx.doi.org/10.1016/j.otohns.2003.12.018

[14] Schlosser, R.J., Woodworth, B.A., Wilensky, E.M., Sean Grady, M. and Bolger, W.E. (2006) Spontaneous Cerebrospinal Fluid Leaks: A Variant of Benign Intracranial Hypertension. Annals of Otology, Rhinology \& Laryngology, 115, 495-500. http://dx.doi.org/10.1177/000348940611500703

[15] Schlosser, R.J. and Bolger, W.E. (2006) Endoscopic Management of Cerebrospinal Fluid Rhinorrhea. Otolaryngologic Clinics of North America, 39, 523-538.

[16] Banks, C., Palmer, J., Chiu, A., O’Malley Jr., B., Woodworth, B. and Kennedy, D. (2009) Endoscopic Closure of CSF Rhinorrhea: 193 Cases over 21 Years. Otolaryngology_Head and Neck Surgery, 140, 826-833. http://dx.doi.org/10.1016/j.otohns.2008.12.060

[17] Carrau, R.L., Snyderman, C.H. and Kassam, A.B. (2005) The Management of Cerebrospinal Fluid Leaks in Patients at Risk for High-Pressure Hydrocephalus. Laryngoscope, 115, 205-212. http://dx.doi.org/10.1097/01.mlg.0000154719.62668.70

[18] Zweig, J.L., Carrau, R.L., Celin, S.E., Schaitkin, B.M., Pollice, P.A., Snyderman, C.H., Kassam, A. and Hegazy, H. (2000) Endoscopic Repair of Cerebrospinal Fluid Leaks to the Sinonasal Tract: Predictors of Success. Otolaryngology-Head and Neck Surgery, 123, 195-201. http://dx.doi.org/10.1067/mhn.2000.107452

[19] Goddard, J.C., Meyer, T., Nguyen, S. and Lambert, P.R. (2010) New Considerations in the Cause of Spontaneous Cerebrospinal Fluid Otorrhea. Otology \& Neurotology, 31, 940-945. http://dx.doi.org/10.1097/MAO.0b013e3181e8f36c

[20] Kutz Jr., J.W., Husain, I.A., Isaacson, B. and Roland, P.S. (2008) Management of Spontaneous Cerebrospinal Fluid Otorrhea. Laryngoscope, 118, 2195-2199. http://dx.doi.org/10.1097/MLG.0b013e318182f833

[21] Silver, R.I., Moonis, G., Schlosser, R.J., Bolger, W.E. and Loevner, L.A. (2007) Radiographic Signs of Elevated Intracranial Pressure in Idiopathic Cerebrospinal Fluid Leaks: A Possible Presentation of Idiopathic Intracranial Hypertension. American Journal of Rhinology, 21, 257-261. http://dx.doi.org/10.2500/ajr.2007.21.3026

[22] Schlosser, R.J. and Bolger, W.E. (2003) Spontaneous Nasal Cerebrospinal Fluid Leaks and Empty Sella Syndrome: A Clinical Association. American Journal of Rhinology, 17, 91-96.

[23] Chaaban, M.R., Illing, E., Riley, K.O. and Woodworth, B.A. (2013) Acetazolamide for High Intracranial Pressure Cerebrospinal Fluid Leaks. International Forum of Allergy \& Rhinology, 3, 718-721. http://dx.doi.org/10.1002/alr.21188

[24] Caballero, N., Bhalla, V., Stankiewicz, J.A. and Welch, K.C. (2012) Effect of Lumbar Drain Placement on Recurrence of Cerebrospinal Rhinorrhea after Endoscopic Repair. International Forum of Allergy \& Rhinology, 2, 222-226. http://dx.doi.org/10.1002/alr.21023

[25] Virk, J.S., Elmiyeh, B. and Saleh, H.A. (2013) Endoscopic Management of Cerebrospinal Fluid Rhinorrhea: The Charing Cross Experience. Journal of Neurological Surgery. Part B, Skull Base, 74, 61-67.

[26] Casiano, R. and Jassir, D. (1999) Endoscopic Cerebrospinal Fluid Rhinorrhea Repair: Is a Lumbar Drain Necessary? Otolaryngology—Head and Neck Surgery, 121, 745-749. http://dx.doi.org/10.1053/hn.1999.v121.a98754 